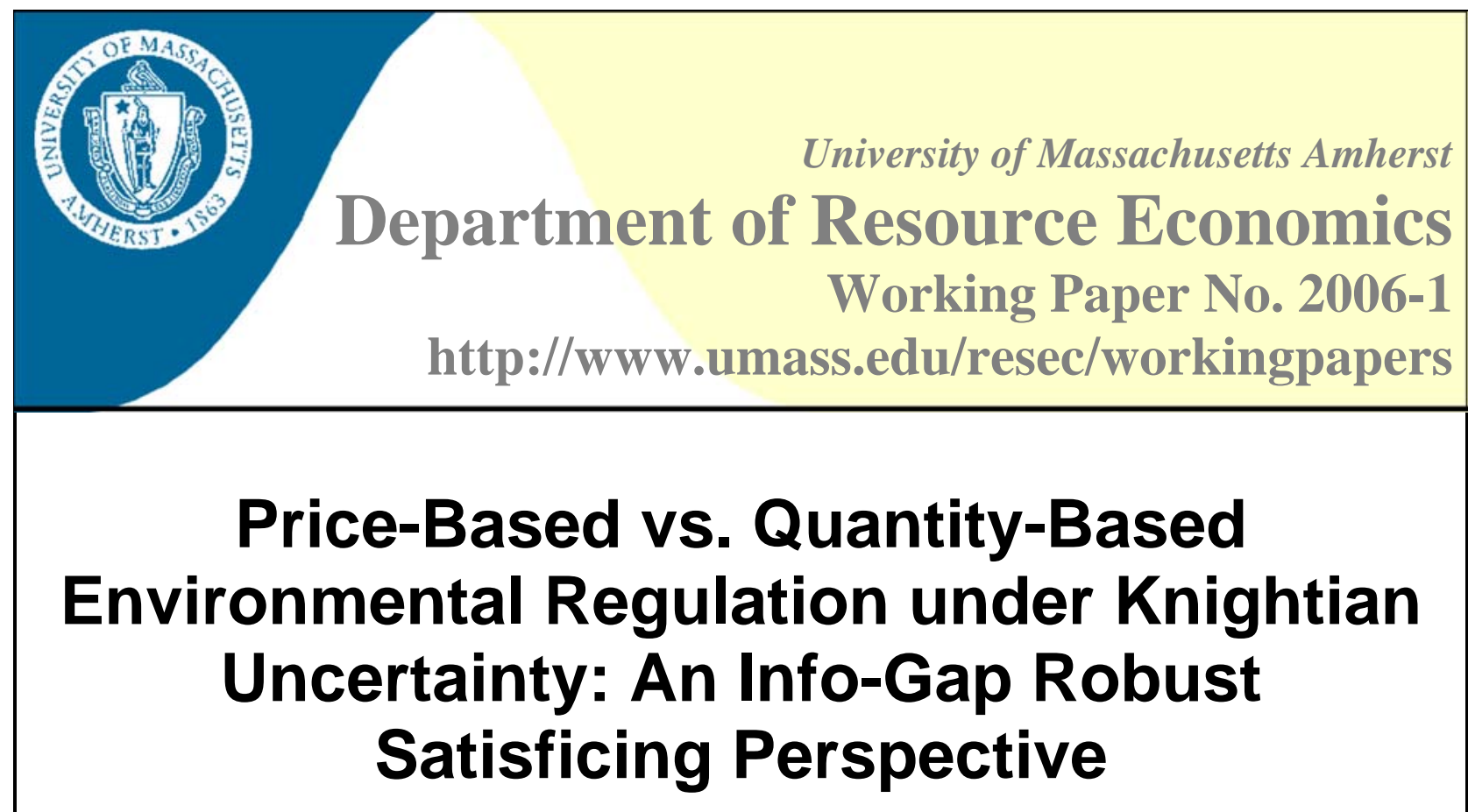

\author{
John K. Stranlund ${ }^{1}$ and Yakov Ben-Haim ${ }^{2}$
}

\begin{abstract}
:
Conventional wisdom among environmental economists is that the relative slopes of the marginal social benefit and marginal social cost functions determine whether a price-based or quantity-based environmental regulation leads to higher expected social welfare. We revisit the choice between price-based vs. quantity-based environmental regulation under Knightian uncertainty; that is, when uncertainty cannot be modeled with known probability distributions. Under these circumstances, the policy objective cannot be to maximize the expected net benefits of emissions control. Instead, we evaluate an emissions tax and an aggregate abatement standard in terms of maximizing the range of uncertainty under which the welfare loss from error in the estimates of the marginal benefits and costs of emissions control can be limited. The main result of our work is that the same criterion involving the relative slopes of the marginal benefit and cost functions determines whether price-based or quantity-based control is more robust to unstructured uncertainty. Hence, not only does the relative slopes criterion lead to the policy that maximizes the expected net benefits of control under structured uncertainty, it also leads to the policy that maximizes robustness to unstructured uncertainty.
\end{abstract}

Keywords: emissions control, environmental regulation, info-gap, Knightian uncertainty, robustness, satisficing

JEL Classification: D81, L51, Q58

\footnotetext{
${ }^{1}$ John K. Stranlund, Department of Resource Economics

University of Massachusetts, 214 Stockbridge Hall, 80 Campus Center Way

Amherst, MA 01003

E: stranlund@resecon.umass.edu P: 413-545-6328 F: 413-545-5853

${ }^{2}$ Yakov Ben-Haim, Mechanical Engineering Technion

Israel Institute of Technology, Haifa 32000 Israel

E: yakov@techunix.technion.ac.il P: 972-4-829-3262 F: 972-4-829-5711
} 
July 2006

\title{
Price-Based vs. Quantity-Based Environmental Regulation under Knightian Uncertainty: An Info-Gap Robust Satisficing Perspective
}

\author{
JOHN K. STRANLUND* \\ Department of Resource Economics \\ University of Massachusetts-Amherst \\ 214 Stockbridge Hall \\ Amherst, MA 01003, USA \\ (413) 545 - 6328 (phone) \\ (413) 545 - 6328 (fax) \\ stranlund@resecon.umass.edu \\ YAKOV BEN-HAIM \\ Yitzhak Moda'i Chair in Technology and Economics \\ Faculty of Mechanical Engineering \\ Technion - Israel Institute of Technology \\ Haifa 32000 Israel \\ yakov@techunix.technion.ac.il
}

\begin{abstract}
Conventional wisdom among environmental economists is that the relative slopes of the marginal social benefit and marginal social cost functions determine whether a price-based or quantity-based environmental regulation leads to higher expected social welfare. We revisit the choice between price-based vs. quantity-based environmental regulation under Knightian uncertainty; that is, when uncertainty cannot be modeled with known probability distributions. Under these circumstances, the policy objective cannot be to maximize the expected net benefits of emissions control. Instead, we evaluate an emissions tax and an aggregate abatement standard in terms of maximizing the range of uncertainty under which the welfare loss from error in the estimates of the marginal benefits and costs of emissions control can be limited. The main result of our work is that the same criterion involving the relative slopes of the marginal benefit and cost functions determines whether price-based or quantity-based control is more robust to unstructured uncertainty. Hence, not only does the relative slopes criterion lead to the policy that maximizes the expected net benefits of control under structured uncertainty, it also leads to the policy that maximizes robustness to unstructured uncertainty.
\end{abstract}

Keywords: emissions control, environmental regulation, info-gap, Knightian uncertainty, robustness, satisficing.

${ }^{*}$ Corresponding author. 


\section{Price-Based vs. Quantity-Based Environmental Regulation under Knightian Uncertainty: An Info-Gap Robust Satisficing Perspective}

\section{Introduction}

Environmental regulations are chosen in a world in which authorities have only imperfect information about the underlying benefits and costs of environmental control. In his seminal paper, Weitzman (1974) provides guidance for the optimal choice between price-based and quantity-based emissions control (i.e., an emissions tax vs. a competitive market for transferable emissions quotas) that has become conventional wisdom among environmental policy scholars and practitioners. Weitzman shows that when the marginal benefits and marginal costs of emissions control are linear (at least to a local approximation), when authorities are uncertain about the intercepts of these functions (but not their slopes), and when the benefits and costs of emissions control are uncorrelated, then a price-based regulation is more efficient than a quantity-based regulation if slope of the marginal cost function is greater than the absolute value of the slope of the marginal benefit function, and a quantity-based regulation dominates if the inequality is reversed. ${ }^{1}$ This decision criterion is determined from a comparison of expected social welfare (expected benefit minus cost) under the two types of regulations.

\footnotetext{
${ }^{1}$ Weitzman (1974) demonstrates that this decision criterion must be modified when benefits and costs are correlated. See Stavins (1996) for a further exposition of this case. Weitzman, Laffont (1977), and Malcomson (1978) examine modifications to the relative-slopes criterion when these are also uncertain. Linearity to a local approximation of the marginal benefit and marginal cost functions is also important for the determination of the prices vs. quantities criterion. Malcomson (1978) and Weitzman (1978) reexamine Weitzman's original work when local linear approximations to the benefit and cost function are not appropriate. In our paper, we stick to linear marginal benefit and cost functions with uncorrelated uncertainty in their intercepts, because it is this problem that drives the conventional wisdom of environmental economists about the choice between emissions taxes and aggregate abatement standards (Baumol and Oates, 1988). In fact, many recent contributions to this literature (e.g., Hoel and Karp, 2002; Moledina et al., 2003; Montero, 2002; Quirion, 2004) assume linear marginal benefit and cost
} 
Weitzman, as well as the small army of scholars who have contributed to the prices vs. quantities debate since 1974, assumed that uncertainty about the marginal benefit and cost functions could be completely characterized by known probability distribution functions over the errors in estimates the marginal benefit and cost functions. ${ }^{2}$ Indeed, the criterion for choosing between price-based and quantity-based regulations is determined from a comparison of expected social welfare under the two regulatory instruments. While it has proven useful to assume that the error structures of estimates of the marginal benefit and cost functions are known, in many cases if not most, environmental authorities must design control policies even when they are not confident of the error structures. That is, the world of environmental decisionmaking may be usefully characterized by Knightian uncertainty in which, not only are the benefit and cost functions unknown, but the distributions of the estimates of these functions are also unknown. ${ }^{3}$

In this paper, we revisit the price vs. quantity debate from the perspective of robust satisficing when the errors in the estimates of the benefits and costs of emissions control are completely unstructured. Info-gap decision theory (Ben-Haim, 2006) provides a useful way to think about the regulatory choice of emissions control instruments in the face of true Knightian

functions with only intercept uncertainty. We note that the approach we take in this paper, that of robust satisficing, can be applied to all of the complicating factors mentioned in this footnote.

${ }^{2}$ Recent contributions to the prices vs. quantities debate in the area of environmental management include extensions to dynamic environments (Hoel and Karp, 2002; Moledina et al., 2003), imperfectly enforced environmental regulations (Montero, 2002), and to economies with pre-existing distortions from labor or capital taxes (Quirion, 2004).

${ }^{3}$ Knight was concerned "with situations which are far too unique, generally speaking, for any sort of statistical tabulation to have any value for guidance. The conception of an objectively measurable probability or chance is simply inapplicable." This is what Knight called "true uncertainty" (Knight, 1921, pp.231-232), and it dominates many economic policy decisions because local or firm-specific details and future contingencies are poorly understood by policymakers. 
uncertainty. ${ }^{4}$ The heart of info-gap analysis is the pursuit of decisions that maximize the range of uncertainty about model parameters or functions within which the decisionmaker is certain to achieve a specified performance criterion. One decision is more robust than another if the range of uncertainty under which the performance criterion is met is larger. ${ }^{5}$ In our case, uncertainty is about the true values of the intercepts of the marginal benefits and costs of emissions control and the performance criterion is to hold the loss associated with error in the estimates of these values to be no greater than some value. For a given loss associated with estimation error, one emissions control policy is more robust than another if the range of error in the estimates of the benefit and cost functions under which the performance criterion is met is larger.

We apply info-gap analysis to the choice between price-based and quantity-based environmental regulation in the conventional model of environmental control under uncertainty. The main result of this paper is that the same criterion involving the relative slopes of the marginal benefit and cost functions that Weitzman derived in the case of structured uncertainty about the estimates of the benefit and cost functions also reveals whether price-based or quantitybased regulation is more robust to this uncertainty when it is completely unstructured. Although we come to the same decision criterion, our motivation for deriving this criterion is very

\footnotetext{
${ }^{4}$ Info-gap decision theory has been applied to a wide variety of problems, including financial risk assessment (Ben-Haim, 2005), search behavior in animal foraging models (Carmel and BenHaim, 2005), policy decisions in marine reserve design (Halpern et al., 2006), natural resource conservation decisions (Moilanen et al., 2006), inspection decisions by port authorities to detect terrorist weapons (Moffitt et al., 2005a) and invasive species (Moffitt et al., 2005b), technological fault diagnosis (Pierce et al., 2006) and engineering model-testing (Vinot et al., 2005).

${ }^{5}$ Info-gap robust-satisficing is a quantitative combination of Knightian uncertainty with Simon's concept of bounded rationality. The robust-satisficing policy-maker seeks a decision which satisfices the performance and is robust to uncertainty. Simon (1983) stresses the importance of satisficing — doing good enough; meeting critical requirements—when decisions must be made with deficient or erroneous information (i.e. Knightian uncertainty) and limited computational resources.
} 
different. While the decision criterion for choosing between price-based and quantity-based emissions control maximizes the expected net social benefits of emissions control, we show that this decision criterion also leads to the choice of policy that is most robust to estimation error when authorities do not have reliable information about the probability structure of this error. Hence, we provide a further justification for the use of the relative-slopes criterion that is conventional wisdom among environmental economists—not only does this criterion lead to the policy that maximizes the expected net benefits of control under structured uncertainty, it also leads to the policy that maximizes robustness to unstructured uncertainty.

The rest of the paper proceeds as follows: In the next section we lay out the structure of the info-gap approach to choosing between a price-based and a quantity-based environmental regulation under true uncertainty. In the third section we calculate the robustness to uncertainty

of each policy type. In the fourth section we derive the condition under which the price approach is more robust to uncertainty than the quantity approach, and vice versa, and discuss the policy significance of our findings. We conclude in the fifth section.

\section{Info-Gap Robust-Satisficing}

\subsection{Info-gap uncertainty about the benefits and costs of pollution control}

Let $q$ denote aggregate reduction (abatement) in the emissions of some pollutant. The aggregate benefits and costs of abatement take on the familiar quadratic forms. The benefit and costs functions are, respectively:

$$
\begin{aligned}
& B(q)=b q-B^{\prime \prime} q^{2} / 2 ; \\
& C(q)=c q+C^{\prime \prime} q^{2} / 2,
\end{aligned}
$$


where $b, c, B^{\prime \prime}$, and $C^{\prime \prime}$ are all positive constants, restricted to guarantee that optimal abatement levels are strictly positive throughout. ${ }^{6}$ The social welfare function is simply the difference between the benefits and costs of abatement:

$$
W(q)=B(q)-C(q)=(b-c) q-\left(B^{\prime \prime}+C^{\prime \prime}\right) q^{2} / 2 .
$$

We assume that the quadratic forms for the benefit and cost functions and the numerical values of $B^{\prime \prime}$ and $C^{\prime \prime}$ have been verified, but that the values of $b$ and $c$ are highly uncertain. Note that $b$ and $c$ are the intercepts of the marginal benefit and marginal cost functions, respectively. Thus, this set-up is consistent with most of the literature on the prices versus quantities debate, in which it is typical to assume that the marginal benefit and cost functions are known except for uncertainty about their intercepts.

Assume that the environmental authority possesses estimates of the intercepts of the marginal benefit and cost functions, denoted $\tilde{b}$ and $\tilde{c}$, but the factors that modify these parameters are complex and poorly understood, so we face true Knightian uncertainty: no reliable probabilistic model is available for the size of the estimation errors. That is, we must deal with the uncertainty in the estimates of these parameters without knowledge of the probability distribution functions that underlie them. Instead, we describe the uncertainty in the estimates of $b$ and $c$ with a fractional-error info-gap model for uncertainty in $\tilde{b}$ and $\tilde{c}$, which is the following unbounded family of nested sets of the marginal functions:

$U(\alpha, \tilde{b}, \tilde{c})=\left\{B^{\prime}(q)=b-B^{\prime \prime} q, C^{\prime}(q)=c+C^{\prime \prime} q:\left|\frac{b-\tilde{b}}{\tilde{b}}\right| \leq \alpha,\left|\frac{c-\tilde{c}}{\tilde{c}}\right| \leq \alpha\right\}, \alpha \geq 0$.

\footnotetext{
${ }^{6}$ Strictly speaking the abatement cost function is the minimum abatement cost function. Implicitly, we assume that abatement responsibilities are allocated among the pollution sources to minimize the aggregate costs of achieving each level of aggregate abatement. This can be achieved with an emissions tax or with a competitive tradable emissions permit policy.
} 
It is clear that $\alpha$ represents the unknown fractional error in the estimates of the intercepts of the marginal benefit and cost functions. When the horizon of uncertainty, $\alpha$, is zero, then the true intercepts, $b$ and $c$, precisely equal the estimated values, $\tilde{b}$ and $\tilde{c}$. The range of possible intercept values increases as the horizon of uncertainty increases. The horizon of uncertainty is unbounded, so there is no known worst case. These properties are characteristic of all info-gap models (Ben-Haim, 2006).

\subsection{Robustness}

Generally, info-gap robustness is the greatest level of uncertainty with which a decision maker is certain to meet a pre-determined performance criterion. In our case, we look for the robustnesses of an emissions tax and an aggregate abatement standard, and our performance criterion is to limit the welfare loss from error in estimating the marginal benefit and cost functions. The emissions tax and the aggregate abatement standard are determined by maximizing the welfare function [3], given the best available estimates of $b$ and $c$. Let the tax be $\tilde{t}$ and the abatement quota be $\tilde{q}$, which are chosen to maximize [3] with $b=\tilde{b}$ and $c=\tilde{c}$. We will calculate $\tilde{t}$ and $\tilde{q}$ in the next section. ${ }^{7}$ The welfare loss incurred with policy $\tilde{p} \in(\tilde{t}, \tilde{q})$, when $b$ and $c$ differ from $\tilde{b}$ and $\tilde{c}$ is denoted $L(\tilde{p}, b, c)$. The policy maker would like low welfare loss, and therefore specifies $L_{c}$ to be the largest acceptable loss. An important result of our analysis will be to evaluate the reliability of attaining any specified level of loss.

\footnotetext{
${ }^{7}$ It is worth noting that $\tilde{t}$ and $\tilde{q}$ are the same price and quantity policies that are derived in conventional analyses of prices vs. quantities. Under the conventional approach to this problem, the expected values of the errors in the intercepts of the marginal benefit and cost functions are assumed to be zero. Maximizing expected welfare then leads to $\tilde{t}$ and $\tilde{q}$.
} 
The robustness-against-uncertainty of policy $\tilde{p}$ is the greatest horizon of uncertainty in the intercepts of the marginal benefit and cost functions (i.e., $\alpha$ ) up to which all realizations of these functions result in loss no greater than $L_{c}$. Formally:

$$
\hat{\alpha}\left(\tilde{p}, L_{c}\right)=\max \left\{\alpha:\left(\max _{b, c \in U(\alpha, \tilde{b}, \tilde{c})} L(\tilde{p}, b, c)\right) \leq L_{c}\right\} .
$$

For policy $\tilde{p}$, we will demonstrate that $\hat{\alpha}\left(\tilde{p}, L_{c}\right)=0$ for $L_{c}=0$. This implies zero

robustness to zero loss; that is, only zero error in the estimates of the $b$ and $c$ can produce zero welfare loss. This is true of any regulation that is based on best estimates of model parameters. Furthermore, we will show that $\hat{\alpha}\left(\tilde{p}, L_{c}\right)$ is strictly increasing in $L_{c}$, which implies that greater robustness to estimation error is only achievable by tolerating a greater loss; that is, robustness to error trades off against loss, and $\hat{\alpha}\left(\tilde{p}, L_{c}\right)$ quantifies this tradeoff.

The main result of this paper is a determination of the relative robustness of the emissions tax, $\tilde{t}$, and the abatement standard, $\tilde{q}$. Specifically, $\tilde{t}$ is more robust to estimation error than $\tilde{q}$ if $\hat{\alpha}\left(\tilde{t}, L_{c}\right)>\hat{\alpha}\left(\tilde{q}, L_{c}\right)$; if the inequality is reversed, the abatement standard is more robust. In the first case, $\hat{\alpha}\left(\tilde{t}, L_{c}\right)>\hat{\alpha}\left(\tilde{q}, L_{c}\right)$ reveals that the range of error in the estimates of the intercept of the marginal benefit and cost function for which an environmental authority is certain that the loss from error will not exceed a critical value, $L_{c}$, is greater under the emissions tax than under the abatement standard. Thus, the authority is more confident about limiting the loss from error under the emissions tax than under the abatement standard. Of course, if $\hat{\alpha}\left(\tilde{t}, L_{c}\right)<\hat{\alpha}\left(\tilde{q}, L_{c}\right)$, then the authority is more confident about limiting the welfare loss from estimation error under the abatement standard. 


\section{Info-Gap Analysis of Price-Based vs. Quantity-Based Environmental Regulation}

In this section we derive the robustness functions for an emissions tax and an aggregate abatement standard, both of which are determined with the best-available estimates of the intercepts of the marginal benefit and cost functions and the info-gap models for uncertainty in those estimates.

\subsection{Robustness of an aggregate abatement standard}

If the intercepts of the marginal benefit and cost functions were known, the environmental authority would choose an aggregate abatement standard to maximize the welfare function

$$
W(q ; b, c)=(b-c) q-\left(B^{\prime \prime}+C^{\prime \prime}\right) q^{2} / 2
$$

which leads to $q^{*}=(b-c) /\left(B^{\prime \prime}+C^{\prime \prime}\right)$. However, with only its best estimates of $b$ and $c$, the authority sets the abatement standard to maximize $W(q ; \tilde{b}, \tilde{c})$, and therefore, sets the abatement standard at $\tilde{q}=(\tilde{b}-\tilde{c}) /\left(B^{\prime \prime}+C^{\prime \prime}\right)$. The welfare loss when $b$ and $c$ differ from $\tilde{b}$ and $\tilde{c}$ is then $L(\tilde{q} ; b, c)=W\left(q^{*} ; b, c\right)-W(\tilde{q} ; b, c)$, which is calculated to be

$$
L(\tilde{q} ; b, c)=[(b-\tilde{b})-(c-\tilde{c})]^{2} / 2\left(B^{\prime \prime}+C^{\prime \prime}\right) .
$$

The uncertainty model [4] specifies the ranges of error in the estimates of the intercepts of the marginal benefit and cost functions at any horizon of uncertainty, $\alpha$; that is, $b-\tilde{b} \in[-\alpha \tilde{b}, \alpha \tilde{b}]$ and $c-\tilde{c} \in[-\alpha \tilde{c}, \alpha \tilde{c}]$. (Recall that $\tilde{b}$ and $\tilde{c}$ are positive constants). The inner maximum of the definition of robustness [5] requires maximization of $L(\tilde{q} ; b, c)$ with respect to $b$ and $c$. In turn, this requires either $b-\tilde{b}=\alpha \tilde{b}$ and $c-\tilde{c}=-\alpha \tilde{c}$, or $b-\tilde{b}=-\alpha \tilde{b}$ and $c-\tilde{c}=\alpha \tilde{c}$. That is, for some fractional error in the estimates of $b$ and $c, \alpha, L(\tilde{q} ; b, c)$ is maximized with 
respect to $b$ and $c$ if $b$ is over-estimated and $c$ is under-estimated, or if $b$ is under-estimated while $c$ is over-estimated. In either case,

$$
L(\alpha, \tilde{q})=\alpha^{2}(\tilde{b}+\tilde{c})^{2} / 2\left(B^{\prime \prime}+C^{\prime \prime}\right),
$$

is the maximum welfare loss of $\tilde{q}$ when the fractional error in the estimates of the intercepts of the marginal benefit and marginal cost functions is $\alpha$.

Now, from [5], the robustness of abatement standard $\tilde{q}$ is the highest $\alpha$ for which $L(\alpha, \tilde{q}) \leq L_{c}$. Since $L(\alpha, \tilde{q})$ is monotonically increasing in $\alpha$, we choose $\alpha$ to satisfy $L(\alpha, \tilde{q})=L_{c}$. Solving this equation for $\alpha$ provides the robustness of the abatement standard $\tilde{q}$ :

$$
\hat{\alpha}\left(\tilde{q}, L_{c}\right)=\sqrt{2 L_{c}\left(B^{\prime \prime}+C^{\prime \prime}\right)} /(\tilde{b}+\tilde{c}) .
$$

Since $\tilde{b}, \tilde{c}, B^{\prime \prime}$, and $C^{\prime \prime}$ are all positive constants and $L_{c}$ is non-negative, $\hat{\alpha}\left(\tilde{q}, L_{c}\right)$ is nonnegative.

The robustness function, $\hat{\alpha}\left(\tilde{q}, L_{c}\right)$, quantifies the greatest fractional error in the estimates of the intercepts of the marginal benefit and marginal cost functions for which the welfare loss associated with the abatement standard $\tilde{q}$ is not greater than some critical welfare loss $L_{c}$. Note that, with respect to $L_{c}, \hat{\alpha}\left(\tilde{q}, L_{c}\right)$ has a zero intercept, is montonically increasing, and strictly concave. The zero intercept implies that there is zero robustness to zero loss; that is, the environmental authority can have no confidence in limiting the loss from estimation error to zero. That robustness is increasing in $L_{c}$ implies that greater robustness only comes with tolerating a greater loss: the authority gains confidence in meeting the performance criterion as this criterion is relaxed. Finally, the concavity of $\hat{\alpha}\left(\tilde{q}, L_{c}\right)$ suggests that the marginal cost (i.e., the marginal increase in critical loss) of attaining greater robustness is increasing. Put another way, confidence in limiting the welfare loss increases with higher acceptable loss, but at a 
decreasing rate. Robustness functions are always monotonic, expressing a fundamental trade-off between robustness and performance. They are not always concave.

\subsection{Robustness of an emissions tax}

We now turn to calculating the robustness of an emissions tax. We demonstrate that it has the same basic characteristics of robustness to an abatement standard.

Firms in an industry will react to an emissions tax, $t$, by choosing their levels of abatement so that their individual marginal abatement cost functions are equal to the tax. In aggregate, then, the intersection of the tax and the aggregate marginal abatement cost function determines aggregate abatement. From $t=c+C^{\prime \prime} q$ we have $q(t)=(t-c) / C^{\prime \prime}$. Social welfare [3], in terms of an emissions tax, is then

$$
W(t ; b, c)=(b-c) q(t)-\left(B^{\prime \prime}+C^{\prime \prime}\right)(q(t))^{2} / 2 .
$$

The tax that maximizes this social welfare function is $t^{*}=\left(b C^{\prime \prime}+c B^{\prime \prime}\right) /\left(B^{\prime \prime}+C^{\prime \prime}\right)$. With only its best estimates of $b$ and $c$, however, the regulatory authority chooses $\tilde{t}=\left(\tilde{b} C^{\prime \prime}+\tilde{c} B^{\prime \prime}\right) /\left(B^{\prime \prime}+C^{\prime \prime}\right)$. (It is easy to demonstrate that $t^{*}$ produces $q^{*}$ abatement, and $\tilde{t}$ produces $\tilde{q}$ abatement). The welfare loss when $b$ and $c$ differ from $\tilde{b}$ and $\tilde{c}$ is $L(\tilde{t} ; b, c)=W\left(t^{*} ; b, c\right)-W(\tilde{t} ; b, c)$, which is calculated to be

$$
L(\tilde{t} ; b, c)=\left[(b-\tilde{b}) C^{\prime \prime}+(c-\tilde{c}) B^{\prime \prime}\right]^{2} / 2\left(B^{\prime \prime}+C^{\prime \prime}\right)\left(C^{\prime \prime}\right)^{2} .
$$

As with the abatement standard, the uncertainty model [4] specifies the ranges of error in the estimates of $b$ and $c, b-\tilde{b} \in[-\alpha \tilde{b}, \alpha \tilde{b}]$ and $c-\tilde{c} \in[-\alpha \tilde{c}, \alpha \tilde{c}]$, at any horizon of uncertainty, $\alpha$. In contrast to the abatement standard, however, the inner maximum of the definition of robustness [5] requires either $b-\tilde{b}=\alpha \tilde{b}$ and $c-\tilde{c}=\alpha \tilde{c}$, or $b-\tilde{b}=-\alpha \tilde{b}$ and $c-\tilde{c}=-\alpha \tilde{c}$. That is, 
$L(\tilde{t} ; b, c)$ is maximized if both $b$ and $c$ are over-estimated, or if both are under-estimated. In either case,

$$
L(\alpha, \tilde{t})=\alpha^{2}\left[\tilde{b} C^{\prime \prime}+\tilde{c} B^{\prime \prime}\right]^{2} / 2\left(B^{\prime \prime}+C^{\prime \prime}\right)\left(C^{\prime \prime}\right)^{2} .
$$

Robustness of $\tilde{t}$ is then the solution to $\arg \max _{\alpha} L(\alpha, \tilde{q}) \leq L_{c}$; that is,

$$
\hat{\alpha}\left(\tilde{t}, L_{c}\right)=C^{\prime \prime} \sqrt{2 L_{c}\left(B^{\prime \prime}+C^{\prime \prime}\right)} /\left(\tilde{b} C^{\prime \prime}+\tilde{c} B^{\prime \prime}\right) .
$$

Again, $\hat{\alpha}\left(\tilde{t}, L_{c}\right)$ is the greatest fractional error in the estimates of the intercepts of the marginal benefit and marginal cost functions for which the welfare loss associated with the emissions tax $\tilde{t}$ is not greater than some critical welfare loss $L_{c}$. With respect to $L_{c}, \hat{\alpha}\left(\tilde{t}, L_{c}\right)$ has a zero intercept, is montonically increasing, and strictly concave. Therefore, $\hat{\alpha}\left(\tilde{t}, L_{c}\right)$ has the same basic structure as $\hat{\alpha}\left(\tilde{q}, L_{c}\right)$. However, for some $L_{c}, \hat{\alpha}\left(\tilde{t}, L_{c}\right)$ and $\hat{\alpha}\left(\tilde{q}, L_{c}\right)$ will normally take on different values.

\section{The Relative Robustness of Price-Based and Quantity-Based Emissions Control}

We are now able to compare the robustness of the emissions tax, $\tilde{t}$, to the robustness of the aggregate abatement standard, $\tilde{q}$. Using the robustness functions [7] and [8], calculate

$$
\hat{\alpha}\left(\tilde{t}, L_{c}\right)-\hat{\alpha}\left(\tilde{q}, L_{c}\right)=\frac{\left(C^{\prime \prime}-B^{\prime \prime}\right) \tilde{c} \sqrt{2 L_{c}\left(B^{\prime \prime}+C^{\prime \prime}\right)}}{(\tilde{b}+\tilde{c})\left(\tilde{b} C^{\prime \prime}+\tilde{c} B^{\prime \prime}\right)} .
$$

Clearly, the relative robustness of $\tilde{t}$ and $\tilde{q}$ to any critical loss, $L_{c}$, depends solely on the relative slopes of the marginal benefit and cost functions, $C^{\prime \prime}$ and $B^{\prime \prime}$. Specifically, $\tilde{t}$ is more robust to estimation error than $\tilde{q}$ if and only if the marginal cost function is more steeply sloped than the marginal benefit function; that is, $C^{\prime \prime}>B^{\prime \prime}$. On the other hand, if $C^{\prime \prime}<B^{\prime \prime}$, the abatement standard is more robust than the emissions tax. Note further that the difference in the 
robustness of the two policies increases as $L_{c}$ increases, Thus, the value of one policy over the other (in terms of immunity to loss) increases as the potential loss $L_{c}$ is allowed to increase. Figures 1 and 2 illustrate the robustness functions for $\tilde{t}$ and $\tilde{q}$ when $C^{\prime \prime}>B^{\prime \prime}$ and $B^{\prime \prime}>C^{\prime \prime}$, respectively. [Place Figures 1 and 2 about here].

The main result of this work is that the same relative-slopes criterion that Weitzman (1974) derived to choose between price-based and quantity-based regulation applies in our case as well. However, we have demonstrated a complementary purpose for this criterion. In the conventional case, the relative-slopes criterion provides guidance about the choice between an emissions tax and an aggregate abatement standard when the policy objective is to maximize expected welfare from emissions control when the errors in the estimates of the intercepts of the marginal benefit and cost functions are characterized by known probability distribution functions. We have shown that the relative-slopes criterion also determines which policy is more robust to unstructured Knightian estimation error. Therefore, our work complements the conventional approach to the prices versus quantities debate, because it provides a further justification for the relative slopes criterion. Not only will this criterion lead to the policy that maximizes the expected net benefits of control under structured uncertainty, it also leads to the policy that maximizes robustness to unstructured uncertainty.

To illustrate the results of our work, suppose that the marginal benefit and cost parameters take on the following values: $\tilde{b}=100, \tilde{c}=40, B^{\prime \prime}=1$, and $C^{\prime \prime}=2$. In this case $\tilde{q}=20$ and $\tilde{t}=80$. Because $C^{\prime \prime}>B^{\prime \prime}, \tilde{t}$ is always more robust that $\tilde{q}$ (Figure 1). To verify this for allowable loss $L_{c}=100$, use [7] to calculate $\hat{\alpha}\left(\tilde{q}, L_{c}=100\right)=0.175$ and use [8] to calculate $\hat{\alpha}\left(\tilde{t}, L_{c}=100\right)=0.204$. That is, welfare loss from estimation error no greater than $L_{c}=100$ is guaranteed with the tax policy if the estimated intercepts err by no more than $\pm 20.4 \%$. In 
contrast, welfare loss no greater than 100 is guaranteed with the quantity policy if the estimated intercepts err by no more than $\pm 17.5 \%$. That is, the robustness premium of the tax policy is \pm 2.9\%. For a higher allowable welfare loss, say $L_{c}=300, \hat{\alpha}\left(\tilde{q}, L_{c}=300\right)=0.303$ and $\hat{\alpha}\left(\tilde{t}, L_{c}=300\right)=0.354$. At this higher level of allowed welfare loss, the robustness premium of the tax policy is $\pm 5.1 \%$. In both of these cases, as well as any $L_{c}>0$, the range of error in the estimates of the intercepts of the marginal benefit and marginal cost functions within which the welfare loss is limited to be no more than $L_{c}$ is greater under the tax than under the abatement standard.

We can use our hypothetical numerical example to illustrate the trade-off between robustness-to-uncertainty and welfare loss, expressed by the positive slopes of the curves in Figures 1 and 2. Whichever policy is chosen, greater robustness can only be obtained in exchange for greater welfare loss. Furthermore, this trade-off is evaluated quantitatively. For instance, a robustness of $\hat{\alpha}=0.2$ corresponds to immunity to $20 \%$ error in the estimated intercepts, $\tilde{b}$ and $\tilde{c}$. For the tax policy, this corresponds to a critical welfare loss of $L_{c}=96$. Pursuing greater robustness, say $40 \%$, requires a greater critical welfare loss of $L_{c}=384$.

\section{Conclusion}

We claim two contributions of this work. First, we have illustrated a policy evaluation tool, infogap robust-satisficing, which is an alternative to expected welfare maximization. Info-gap robust-satisficing is useful when errors in model parameters are unstructured in the sense that they cannot be modeled with known or confidently estimated probability distribution functions. In these cases, one cannot evaluate policies in terms of their affect on expected benefits and 
costs. Info-gap robust satisficing requires no knowledge of underlying probability models, and evaluates policies on the basis of robustness to loss when uncertainty is unstructured. Our second contribution is that we have reinterpreted the conventional wisdom of environmental economists that the relative slopes of the marginal benefits and marginal costs of pollution control determine whether price-based or quantity-based policies should be pursued. It is conventional wisdom that the relative-slopes criterion leads to the policy type that maximizes the expected net social benefits of environmental control under structured uncertainty about social benefits and costs. We have shown that this criterion also leads to the policy choice that is more robust to unstructured uncertainty about social benefits and costs.

We applied the info-gap robust satisficing approach to the canonical model of environmental control under uncertainty that drives the conventional wisdom about prices versus quantities. It is well-known, however, that the relative-slopes criterion must be modified when there is also uncertainty in the slopes of the marginal benefit and cost functions, when the uncertainty in benefits and costs are correlated, when the marginal benefit and cost functions are nonlinear, as well as when many of the other simplifying assumptions that underlie the conventional model are relaxed. (See footnotes 1 and 2). The approach we take in this paper can easily be applied to all of these more complicated situations. We believe that doing so would be a fruitful area for future research, and will lead to a deeper understanding of the design of environmental policies under uncertainty, and possibly to different policy recommendations. 


\section{Acknowledgements:}

Professor Stranlund gratefully acknowledges funding for this research by the U. S. Department of Agriculture under USDA/ERS/PREISM Cooperative Agreement No. 43-3AEM-4-80115, and the Cooperative State Research Extension, Education Service, U. S. Department of Agriculture, Massachusetts Agricultural Experiment Station under Project No. MAS00861. 


\section{References}

Baumol, William J. and Wallace E. Oates. 1988. The Theory of Environmental Policy, Cambridge University Press.

Ben-Haim, Yakov. 2006. Info-Gap Decision Theory: Decisions Under Severe Uncertainty. Second Edition, Academic Press.

Ben-Haim, Yakov. 2005. "Value at risk with info-gap uncertainty.” Journal of Risk Finance 6(5), 388-403.

Carmel, Yohay and Yakov Ben-Haim. 2005. "Info-gap robust-satisficing model of foraging behavior: Do foragers optimize or satisfice?” American Naturalist 166(5), 633-641.

Halpern, Benjamin S.; Helen M. Regan, Hugh P. Possingham and Michael A. McCarthy. 2006. “Accounting for uncertainty in marine reserve design.” Ecology Letters 9, 2-11.

Hoel, Michael and Larry Karp. 2002. “Taxes versus quotas for a stock pollutant.” Resource and Energy Economics 24(4) 367-384.

Knight, Frank H. 1921. Risk, Uncertainty and Profit. Houghton Mifflin Co. Re-issued by University of Chicago Press, 1971.

Laffont, Jean Jacques. 1977. “More on prices vs. quantities.” Review of Economic Studies 44(1), 177-182.

Malcomson, James. 1978. "Prices vs. quantities: A critical note on the use of approximations." Review of Economic Studies 45(1), 203-207.

Moffitt, L. Joe; John K. Stranlund and Barry C. Field. 2005a. "Inspections to avert terrorism: Robustness under severe uncertainty.” Journal of Homeland Security and Emergency Management 2(3), Article 3. http://www.bepress.com/jhsem/vol2/iss3/3.

Moffitt, L. Joe; John K. Stranlund, Barry C. Field, and Craig D. Osteen. 2005b. "Robust inspection for invasive species with a limited budget." Forthcoming in The Economics of Plant Health. Alfons Oude Lansink (ed.). Springer.

Moilanen, Atte and Brendan A. Wintle. 2006. "Uncertainty analysis favours selection of spatially aggregated reserve structures.” Biological Conservation 129(3), 427-434.

Moledina, Amyaz A.; Jay S. Coggins, Stephen Polasky and Christopher Costello. 2003. "Dynamic environmental policy with strategic firms: Prices versus quantities." Journal of Environmental Economics and Management 45(2S), 356-376.

Montero, Juan-Pablo. 2002. “Prices versus quantities with incomplete enforcement.” Journal of Public Economics 85: 435-454.

Pierce, S.G.; K. Worden and G. Manson. 2006. “A novel information-gap technique to assess reliability of neural network-based damage detection.” Journal of Sound and Vibration 293(1-2), 96-111.

Quirion, Phillippe. 2004. "Prices versus quantities in a second-best setting.” Environmental \& Resource Economics 29(3): 337-359.

Simon, Herbert A. 1983. Reason in Human Affairs. Stanford University Press, Stanford. 
Stavins, Robert. 1996. “Correlated uncertainty and policy instrument choice.” Journal of Environmental Economics and Management 30(2), 218-232.

Vinot, P.; S. Cogan and V. Cipolla. 2005. “A robust model-based test planning procedure.” Journal of Sound and Vibration 288(3), 571-585.

Weitzman, Martin. 1974. "Prices vs. quantities.” Review of Economic Studies 41(4), 477-491.

Weitzman, Martin. 1978. "Reply to "Prices vs. quantities: A critical note on the use of approximations” by James M. Malcomson.” Review of Economic Studies 45(1), 209-210. 


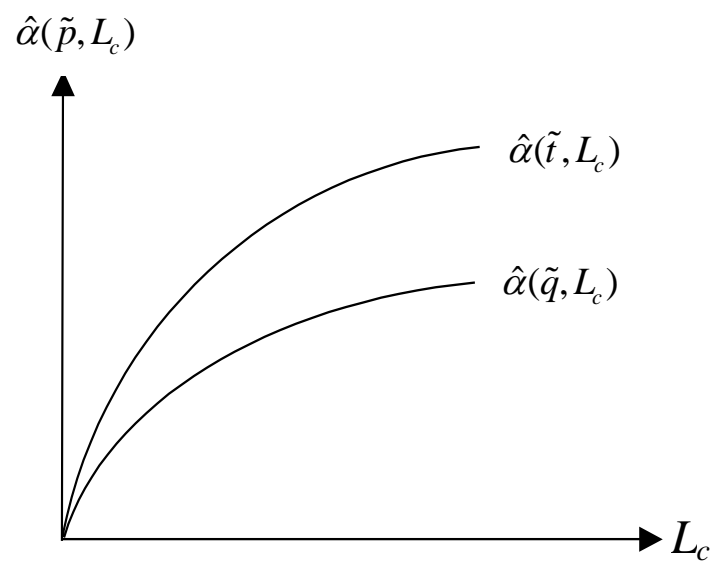

Figure 1: Relative robustness of $\tilde{t}$ and $\tilde{q}$ when $C^{\prime \prime}>B^{\prime \prime}$.

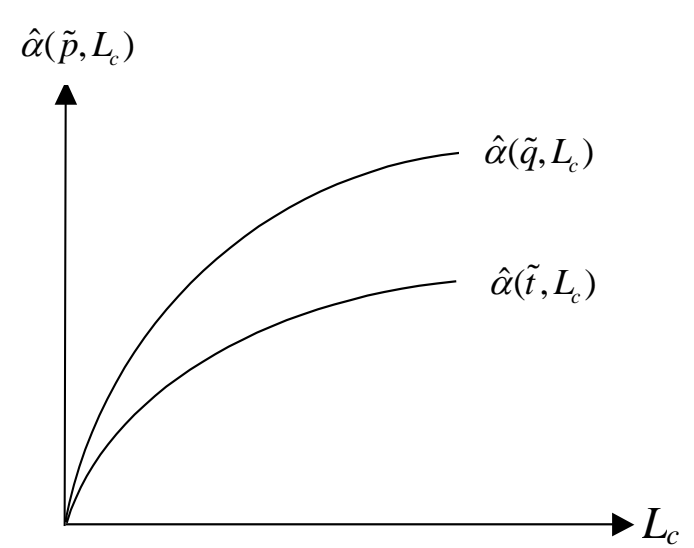

Figure 2: Relative robustness of $\tilde{t}$ and $\tilde{q}$ when $B^{\prime \prime}>C^{\prime \prime}$. 